PoS $\quad \begin{aligned} & \text { PROCEEDINGS } \\ & \text { OF SCIENCE }\end{aligned}$

\title{
Stopping, particle production and Upsilon suppression in heavy-ion collisions at the LHC
}

\author{
G. Wolschin* \\ Institut für Theoretische Physik der Universität Heidelberg, Philosophenweg 16, \\ D-69120 Heidelberg, Germany, EU \\ E-mail: wolschin@uni-hd.de
}

\begin{abstract}
Three characteristic features of relativistic heavy-ion collisions are considered: (1) Stopping at RHIC and LHC energies in a QCD-based model, (2) Charged-hadron production in a relativistic diffusion model (RDM), and (3) Bottomium suppression in $\mathrm{PbPb}$ collisions at the current $\mathrm{LHC}$ energy of $\sqrt{s_{N N}}=2.76 \mathrm{TeV}$.
\end{abstract}

The XXI International Workshop High Energy Physics and Quantum Field Theory, June 23 âĂS June 30, 2013

Saint Petersburg Area, Russia

\footnotetext{
* Speaker.
} 


\section{Introduction}

In this article I consider three different features of relativistic heavy-ion collisions in theoretical approaches that are adapted to the individual problem. Starting with the initial phase of a heavy-ion collision, I first discuss the problem of stopping at SPS, RHIC and LHC energies. Due to the gluon condensate that becomes more and more important at higher energies, this is mainly dealt with in a QCD-based partonic model of valence quark - gluon interactions, although hadronic processes in the baryonic transport contribute at lower (SPS) energies, as a close comparison with net-proton (proton minus antiproton) SPS data reveals.

The next facet of relativistic heavy-ion collisions considered here is the production of charged hadrons in a nonequilibrium-statistical relativistic diffusion model (RDM), which I had earlier developed for and applied to stopping, but it actually yields more precise results for particle production due to the larger statistical ensemble. Particular emphasis is on the relative importance of the fragmentation sources as compared to the mid-rapidity gluon-gluon source (which is absent in net-baryon distributions because produced particles and their antiparticles cancel out in g-g). It turns out that the total particle content of the gluon-gluon source becomes more important than that of the fragmentation sources as the energy increases from RHIC to LHC. The centrality dependence is well described, and predictions for the LHC design energy of $5.52 \mathrm{TeV}$ in $\mathrm{PbPb}$ become possible.

As an example for the suppression of heavy quarkonia in the quark-gluon plasma in $\mathrm{PbPb}$ collisions with respect to $p p$ at the same LHC energy of $2.76 \mathrm{TeV}$, I consider the heavy $\Upsilon$ meson in its $1 S$ ground state, and in the $2 S$ and $3 S$ excited states that have been measured very precisely by the CMS collaboration - one of the most spectacular results of heavy-ion physics at the LHC. In a model that encompasses the dissociation of these states in the thermal gluon environment, their collisional damping, and the screening of the real part of the quark-antiquark potential for the excited states together with the modification of the feed-down cascade due to QGP formation, the centrality-dependent suppression of the ground state is well understood, whereas additional effects need to be considered for the excited-state suppression.

\section{Stopping in heavy-ion collisions at SPS, RHIC and LHC energies}

Baryon stopping in central heavy-ion collisions at low relativistic energies in the AGS and SPS region is well described in a relativistic diffusion model that allows to calculate analytically netbaryon rapidity distributions based on non-equilibrium-statistical considerations [1]. However, it does not offer a direct indication whether the physical origin of stopping is of hadronic or partonic nature.

In a partonic QCD-based model such as $[2,3]$ that is tailored to high-energy processes one should, however, expect deviations from the data in certain net-baryon observables when the energy is reduced and hence, hadronic processes become important. Predictions of this partonic approach have therefore been compared to the available data in order to describe stopping, and find signatures for the transition from soft hadronic to hard partonic processes in the corresponding net-baryon (proton) observables such as rapidity distributions. 


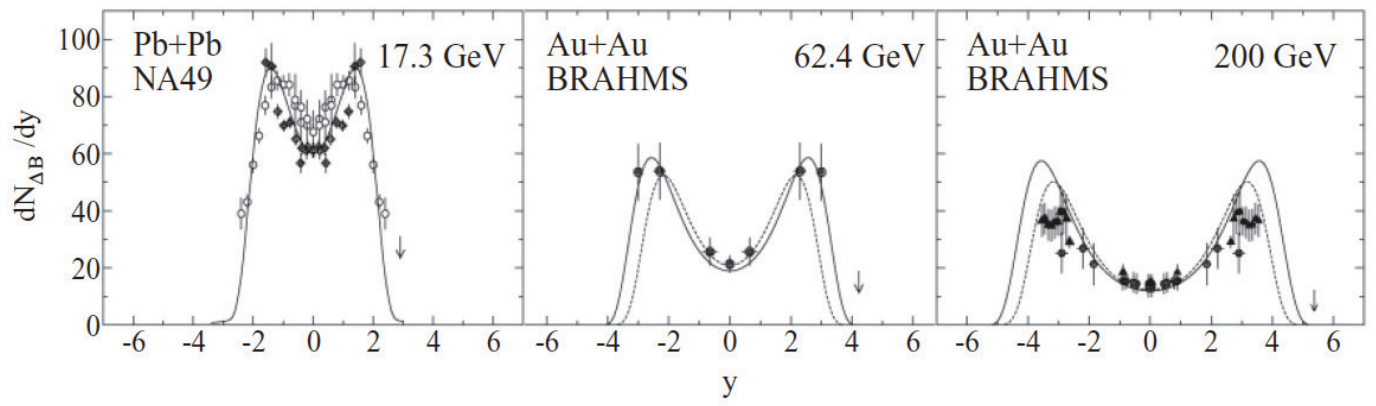

Figure 1: Calculated rapidity distribution of net baryons in a QCD-based approach to describe stopping in comparison with SPS and RHIC data. Central $\mathrm{Pb}+\mathrm{Pb}$ collisions at SPS energies of $\sqrt{s_{N N}}=17.3 \mathrm{GeV}$ (left, NA49 data [4, 5]), and AuAu at RHIC energies of $62.4 \mathrm{GeV}$ (middle), $200 \mathrm{GeV}$ (right). BRAHMS data are from $[6,7,8,9]$. The theoretical fragmentation peak position depends weakly on the gluon saturation scale $Q_{s}$, see [3] for details. Theoretical distributions are shown here for values of the saturation-scale exponent $\lambda=0.2$ (solid curves). Arrows indicate the beam rapidities. Dotted curves include the effect of a fragmentation function. From Mehtar-Tani and Wolschin [3].

We have investigated in $[2,3,10,11]$ both the mean rapidity loss $\langle\delta y\rangle$, and the fragmentation peak position $y_{\text {peak }}$ as functions of the beam rapidity $y_{\text {beam }}$ (or center-of-mass energy $\sqrt{s_{N N}}$ ). Already at a SPS energy of $\sqrt{s_{N N}}=17.3 \mathrm{GeV}$, the fragmentation peaks are clearly visible in the NA49 data [4], see figure 1 [3]. They move further apart in rapidity space as the energy is increased to 62.4 GeV and $200 \mathrm{GeV}$ at RHIC. At the maximum LHC energy of $5.52 \mathrm{TeV}$, the fragmentation peaks are even further separated, and expected to be about 2 units of rapidity below the beam value according to the microscopic prediction, see figure 2 [2] $\left(y_{\text {beam }}=8.68\right)$.

Most of the physical processes leading to the rapidity loss in the peak region are of partonic nature: Fast valence quarks in the projectile collide with the gluon condensate in the respective other beam, thereby exchanging soft gluons, and reducing the beam energy and rapidity. This behaviour should persist also at lower incident energies such as those reached at RHIC and SPS, and one expects a linear dependence of the peak position (or the rapidity loss from $y_{\text {beam }}$ to $y_{\text {peak }}$ ) on the beam rapidity.

In contrast, the mean rapidity loss that involves physical processes over the whole range from midrapidity to the beam value is not only due to partonic events, but will gradually involve hadronic energy-loss mechanisms. The expected indication for this effect are deviations of the mean rapidity $\operatorname{loss}\langle\delta y\rangle$ from a linear dependence on the beam rapidity.

Results for the fragmentation peak position as function of the beam rapidity as extracted [10] from NA49 [5] and BRAHMS [6] data are shown in figure 3 [10]. They fall on a straight line which agrees exactly with the analytical prediction of our partonic model based on gluon saturation [2]

$$
y_{\text {peak }}=\frac{1}{1+\lambda}\left(y_{\text {beam }}-\ln A^{1 / 6}\right)+\text { const }
$$

for a saturation-scale exponent $\lambda=0.2$, an empirical const $=-0.2$, and the mass number $A$. Here $\lambda$ determines the Bjorken- $x$ dependent value of the gluon saturation momentum $Q_{s}^{2}=A^{1 / 3} Q_{0}^{2} x^{-\lambda}$, with $Q_{0}^{2} \simeq 0.04 \mathrm{GeV}^{2}$ setting the momentum scale. The saturation-scale exponent $\lambda=0.2$ corresponds to a gluon saturation momentum of $Q_{s} \simeq 0.77 \mathrm{GeV}$ for $\mathrm{A}=208$ at $x=0.01$. 


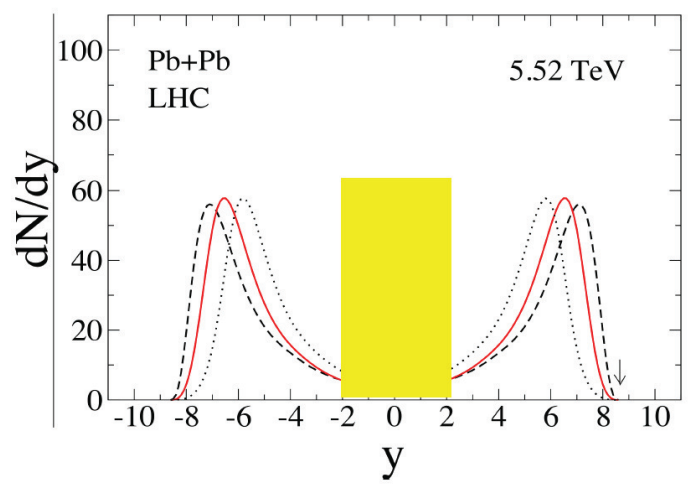

Figure 2: Rapidity distribution of net baryons in central $\mathrm{Pb}+\mathrm{Pb}$ collisions at LHC energies of $\sqrt{s_{N N}}=5.52$ $\mathrm{TeV}$. The fragmentation peak position depends considerably on the gluon saturation scale $Q_{s}$. Theoretical distributions are shown for values of the saturation-scale exponent $\lambda=0$ (dashed), $\lambda=0.15$ (solid), and $\lambda=0.3$ (dotted curve) [2]. ALICE currently provides particle identification only in the shaded midrapidity region, the peaks are experimentally not yet accessible. From [2], inset from [11].

The predicted linear growth of $y_{\text {peak }}$ with $y_{\text {beam }}$ is found to be valid also at low SPS energies, see figure 3. This indicates that even in this region of low relativistic energies, the processes that lead to an energy and rapidity loss in the peak region are predominantly partonic.

The mean rapidity loss is obtained by subtracting the average rapidity from the beam rapidity, $\langle\delta y\rangle=y_{\text {beam }}-\frac{2}{N_{\text {part }}} \int_{0}^{y_{\text {beam }}} y \frac{d N_{B-\bar{B}}}{d y} d y$. It reflects the properties of the system over the whole rapidity range, not just at the peak position, and may therefore show a qualitatively different dependence on the beam rapidity $y_{\text {beam }}$ (or the initial c.m. energy $\sqrt{s_{N N}}$ ) than the fragmentation-peak position $y_{\text {peak }}$. Indeed it is found $[12,9,2]$ to deviate from a simple straight-line dependence on the beam rapidity, see figure 4 [3].

In the high energy limit, the partonic model based on the concept of gluon saturation [2] predicts for the mean rapidity loss $\langle\delta y\rangle=\frac{\lambda}{1+\lambda} y_{\text {beam }}+$ const $^{\prime}$. This result is indicated by the straight line in figure 4 for $\lambda=0.2$ [11]. The limit should be reached in $\mathrm{PbPb}$ collsions at the highest LHC energies. At lower energies, the data for the mean rapidity loss (figure 4) clearly show an increasing deviation that is indicative for the gradual onset of hadronic processes that are not accounted for in the partonic high-energy limit, and thus, for the increasing relevance of confinement.

\section{Hadron production in heavy-ion collisions at LHC energies}

Most theoretical models for particle production in relativistic heavy-ion collisions focus on gluon-gluon production (see [14] as an example). The particle and antiparticle distributions are then identical, which is not the case experimentally, see for example in $\pi^{+}$and $\pi^{-}$distribution functions [15]. Hence, the fragmentation sources from quark-gluon interactions have to be considered in addition. In net-baryon distributions (baryons minus antibaryons) the gluon-gluon source that is peaked at midrapidity even cancels out such that only the fragmentation sources remain $[2,3]$, giving rise to two fragmentation peaks that are clearly seen in the data at high SPS and RHIC energies, and in the theoretical predictions at LHC energies, see the previous chapter. 


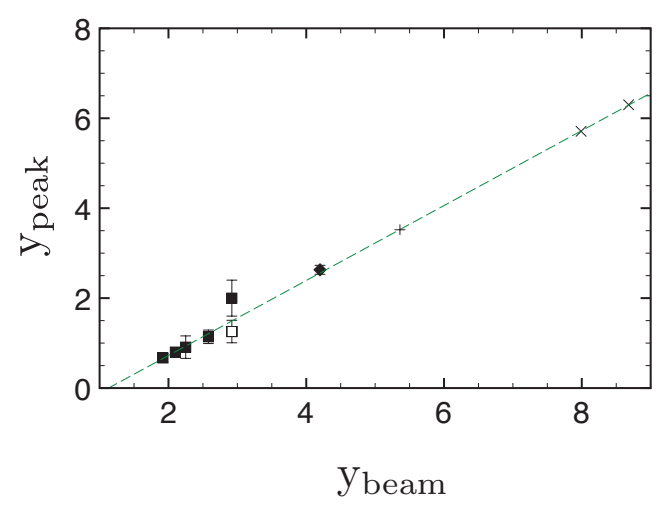

Figure 3: Peak positions of the net-proton rapidity distributions in $\mathrm{Pb}+\mathrm{Pb}$ (black squares) and $\mathrm{Au}+\mathrm{Au}$ (black diamond) as function of the beam rapidity, determined from double-gaussian fits [10] of the NA49 $[13,5]$ and RHIC data [9]. The open square is based on older NA49 data at $17.3 \mathrm{GeV}$ [4]. The slope agrees well with the analytical expression eq.(2.1) for $\lambda=0.2$, dashed line. The cross refers to the calculated peak position of $\mathrm{Au}+\mathrm{Au}$ at $200 \mathrm{GeV}$, the inclined crosses to $\mathrm{Pb}+\mathrm{Pb}$ at LHC energies of 2.76 and $5.52 \mathrm{TeV}$. The data show no deviation from the straight line, indicating the dominance of partonic processes at the peak position. From [10].

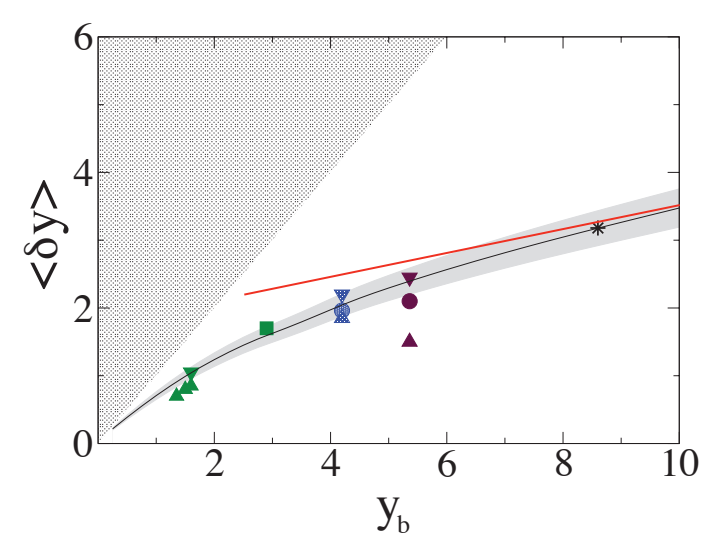

Figure 4: The mean rapidity loss $\langle\delta y\rangle$ as obtained in [3] is plotted as a function of beam rapidity $y_{\text {beam }}$, solid curve. The star at $y_{\text {beam }}=8.68$ is the prediction for central $\mathrm{Pb}+\mathrm{Pb}$ at LHC-energies of $\sqrt{s_{N N}}=5.52 \mathrm{TeV}$ with $\lambda=0.2$. Analysis results from AGS Au + Au data (E917, E802/E866, triangles) [12], SPS Pb $+\mathrm{Pb}$ data (NA49, square [4]), RHIC Au + Au data (BRAHMS, dots, with triangles as lower and upper limits [6, 9]) are compared with the calculations $[2,3]$. The solid straight line is the analytical prediction of the slope in the high-energy partonic limit for $\lambda=0.2$. Below the highest RHIC energies there is a clear deviation from the partonic limit due to the gradual onset of hadronic processes - as a consequence of confinement in the mean rapidity loss. No such deviation appears in the corresponding fragmentation peak positions, see figure 3. From [3], inset from [11]. 
The effect of the fragmentation sources is less obvious for produced particles (rather than net baryons), but it has to be considered in theoretical treatments. In [16] I have proposed to investigate the relative importance of gluon-gluon vs. fragmentation sources as a function of c.m. energy in collisions of heavy systems ( $\mathrm{AuAu}, \mathrm{PbPb})$ using a relativistic diffusion model (RDM) [17, 18], which is a phenomenological nonequilibrium-statistical model. It has proven to be useful in the analysis of data and in predictions for asymmetric [19] and symmetric [20] systems. Its three sources correspond to the gluon-gluon and fragmentation sources of the available microscopic theories. The relative sizes of these underlying components can be determined in direct comparisons of the RDM sources with the data as functions of the incident energy.

The gluon-gluon source is unimportant in charged-hadron production at SPS and low RHIC energies up to $\sqrt{s_{N N}} \simeq 20 \mathrm{GeV}$ [21], and the measured pseudorapidity distributions are well reproduced from the fragmentation sources only. Here the fragmentation sources are peaked close to midrapidity. They are consequently strongly influenced by the Jacobian transformation from rapidity to pseudorapidity space. At higher energies, the fragmentation peaks move apart, the central gluon-gluon source emerges, and the Jacobian increasingly affects only the central source. Also, its overall effect becomes smaller with rising energy since it depends on $\left(\langle m\rangle / p_{T}\right)^{2}$, but a consideration of the Jacobian is still essential for the accurate calculation of pseudorapidity distributions at LHC energies. The pronounced midrapidity dip seen in the ALICE PbPb charged-hadron data is thus caused by the interplay of fragmentation and central sources, plus the effect of the Jacobian on the central source.

A brief outline of the method used to determine the relative size and extent of the sources in $\eta$-space is given in the next section [16]. Results for heavy systems at RHIC and LHC energies are presented, and the energy dependence of central and fragmentation sources is discussed. Conclusions are drawn in section 5.

\subsection{RDM with three sources}

In [16] and references therein, the rapidity distributions of produced particles are calculated in the three-sources version of the relativistic diffusion model from an incoherent superposition of the fragmentation sources $R_{1,2}\left(y, t=\tau_{\text {int }}\right)$ with charged-particle content $N_{c h}^{1}$ (projectile-like), $N_{c h}^{2}$ (target-like) and the gluon-gluon source $R_{g g}\left(y, t=\tau_{\text {int }}\right)$ with charged-particle content $N_{c h}^{g g}$ as

$$
\frac{d N_{c h}\left(y, t=\tau_{\text {int }}\right)}{d y}=N_{c h}^{1} R_{1}\left(y, \tau_{\text {int }}\right)+N_{c h}^{2} R_{2}\left(y, \tau_{\text {int }}\right)+N_{c h}^{g g} R_{g g}\left(y, \tau_{\text {int }}\right)
$$

with the rapidity $y=0.5 \cdot \ln ((E+p) /(E-p))$, and the interaction time $\tau_{\text {int }}$ (total integration time of the underlying partial differential equation). In the linearized RDM [17], the macroscopic distribution functions are solutions of the Fokker-Planck equation $(k=1,2,3)$

$$
\frac{\partial}{\partial t} R_{k}(y, t)=-\frac{1}{\tau_{y}} \frac{\partial}{\partial y}\left[\left(y_{e q}-y\right) \cdot R_{k}(y, t)\right]+D_{y}^{k} \frac{\partial^{2}}{\partial y^{2}} R_{k}(y, t)
$$

Integrating the equation with the initial conditions $R_{1,2}(y, t=0)=\delta\left(y \pm y_{\max }\right)$, the absolute value of the beam rapidities $y_{\max }$, and $R_{3=g g}(y, t=0)=\delta\left(y-y_{e q}\right)$ yields the exact solution as described in [16], and references therein. 


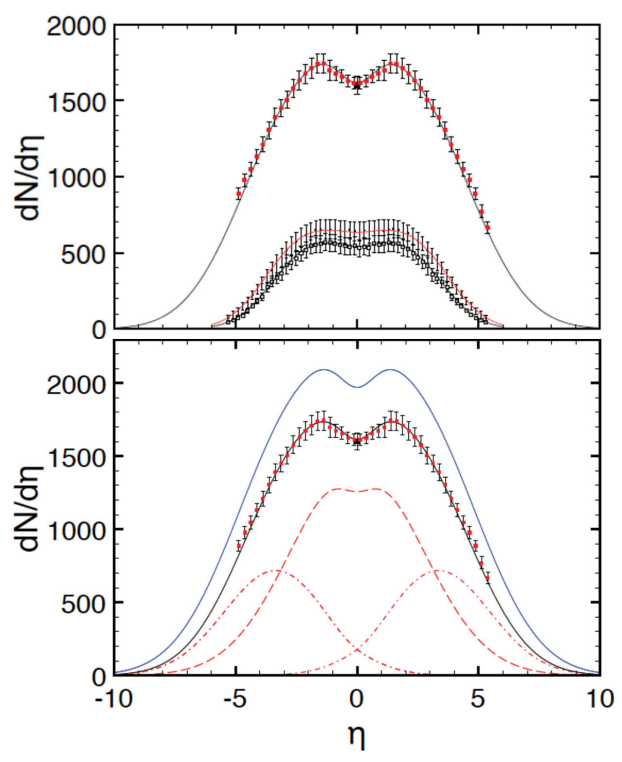

Figure 5: The RDM pseudorapidity distribution function for charged hadrons in central $\mathrm{PbPb}$ collisions at LHC energies of $2.76 \mathrm{TeV}$, and central AuAu at RHIC energies of 130 and $200 \mathrm{GeV}$ with RDM parameters [16] adjusted to the PHOBOS [22] and ALICE [23, 24], upper frame. In the bottom frame, the underlying theoretical distributions are shown for $2.76 \mathrm{TeV} \mathrm{PbPb}$. The shape of the midrapidity source is modified by the Jacobian. At LHC energies, the midrapidity value is mostly determined by particle production from gluon-gluon collisions. The upper curve is the RDM-prediction for 5.52 TeV. From [16].

Pseudorapidity distributions of produced charged particles are then obtained by converting the calculated distribution functions to pseudorapidity space, $\eta=-\ln [\tan (\theta / 2)]$. The well-known Jacobian transformation

$$
\frac{d N}{d \eta}=\frac{d N}{d y} \frac{d y}{d \eta}=\frac{d N}{d y} \cosh (\eta) \cdot\left[1+\left(m / p_{T}\right)^{2}+\sinh ^{2}(\eta)\right]^{-1 / 2}
$$

depends on the squared ratio of the mass and the transverse momentum of the produced particles: Its effect increases with the mass of the particles, and it is most pronounced at small transverse momenta. For reliable results one has to consider the full $p_{T}$-distribution, however. In $[20,16]$ it is outlined how this can be done approximately.

In the fragmentation region where LHC data are not available, we have proposed in [20] to use the well-known limiting fragmentation scaling hypothesis [25] as an additional constraint. We use 0.2 TeV AuAu results at RHIC - where data in the fragmentation region are available - to supplement the $\mathrm{LHC} 2.76 \mathrm{TeV} \mathrm{PbPb}$ data in analogous centrality classes at large values of pseudorapidity as described in [20].

\subsection{Results for hadron production}

The RDM result for the pseudorapidity distribution of produced charged hadrons $2.76 \mathrm{TeV}$ $\mathrm{PbPb}$ is shown in figure 6 [16] together with recent ALICE data [24] for $0-5 \%$ centrality in a $\chi^{2}$ optimization. Parameters are given in [16]. 


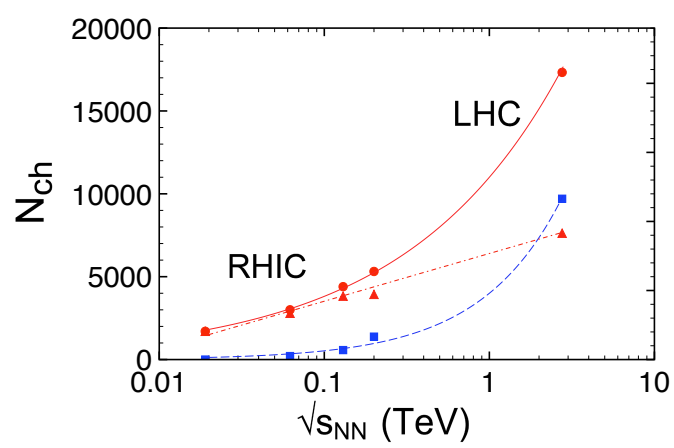

Figure 6: Number of produced charged hadrons as function of the c.m. energy $\sqrt{s_{N N}}$ from RDM-fits of the available data for central heavy-ion collisions at 0.019, 0.062, 0.13, $0.2 \mathrm{TeV}$ (RHIC, AuAu), and $2.76 \mathrm{TeV}$ $(\mathrm{LHC}, \mathrm{PbPb})$. Circles are the total numbers, squares are hadrons produced from the midrapidity source, and triangles are particles from the fragmentation sources. The gluon-gluon source (dashed) becomes the main source of particle production between RHIC and LHC energies. From [16].

The relative size of the three sources in central $2.76 \mathrm{TeV} \mathrm{PbPb}$ is displayed in the lower frame of figure 5. The midrapidity source already contains the largest fraction of produced charged hadrons. Its shape is significantly deformed by the Jacobian transformation from rapidity to pseudorapidity space, whereas the fragmentation sources are not much influenced by the transformation.

The midrapidity dip is more pronounced at LHC energies as compared to RHIC energies, although the effect of the Jacobian tends to be smaller at the higher incident energy. This clearly indicates that there has to be a physical origin of the midrapidity dip in addition to the effect of the Jacobian, and in [16] I have proposed that the interplay of the three sources provides the observed effect. In $2.76 \mathrm{TeV} \mathrm{PbPb}$ collisions, the fragmentation sources are peaked at large values $\left.\left(<y_{1,2}\right\rangle=3.34\right)$ of rapidity - whereas at $0.2 \mathrm{TeV}$ RHIC energy, the center is at $\left\langle y_{1,2}\right\rangle=2.4$. Consequently, the midrapidity yield at LHC energies is essentially due to the central source, with only a small contribution from the fragmentation sources. Although the relative particle content in the central source is larger at LHC energies than at RHIC, this produces the observed midrapidity dip, together with the effect of the Jacobian on the central source.

\subsection{Energy dependence of the three sources}

Charged-hadron production at RHIC [22] and LHC [24] energies has been investigated experimentally in sufficient detail to provide conclusions regarding the relative size of the three particle production sources as function of energy in heavy-ion collisions (AuAu at RHIC, $\mathrm{PbPb}$ at $\mathrm{LHC}$ ). The energy dependence of the sources is displayed in figure 6, with parameters given in [16]. Here the total charged-hadron production (circles) follows a power law $\propto s_{N N}^{0.23}$. The hadrons produced from the central source (squares) have an even stronger dependence on initial energy according to $\propto s_{N N}^{0.44}$, whereas particles produced in the fragmentation sources have a weaker dependence $\propto \log \left(s_{N N} / s_{0}\right)$.

The rising particle production yield from the central (gluon-gluon induced) source with incident energy is due to the increasing gluon content of the system at high relativistic energies. 


\section{Bottomium suppression in PbPb collisions at $\mathrm{LHC}$ energies}

In relativistic heavy-ion collisions the suppression of quarkonium $(Q \bar{Q})$ states as compared to the yield in $p p$ collisions at the same energy (scaled with the number of binary collisions) is an interesting probe for the investigation of the quark-gluon plasma that is likely produced in sufficiently central collisions at RHIC and LHC energies.

Whereas charm quarks are produced abundantly at LHC energies such that statistical regeneration effects become important in case of $J / \Psi$ suppression, only few of the three times heavier bottom quarks are produced, and the bottomium system becomes a cleaner probe than charmonium, also from the theoretical point of view. Its $\Upsilon(1 S)$ ground state is very strongly bound and therefore not sensitive to the screening $[26,27,28,29]$ of the real part of the quark-antiquark potential except at temperatures larger than 4.1 times the critical temperature (which we take here as $T_{c} \simeq 170 \mathrm{MeV}$.

Hence, the direct suppression of the $\Upsilon(1 S)$ state in the QGP medium is mostly caused by the imaginary part of the quark-antiquark potential that accounts for collisional damping, and by gluodissociation through the dipole interaction with thermal gluons, see [30], and references therein. In addition, since the suppression of excited states through screening, damping and gluodissociation is large, the correspondingly reduced feed-down to the ground state needs to be carefully taken into account. The suppression of the $\Upsilon(1 S)$ ground state (mass $9.46 \mathrm{GeV}$ ) down to $56 \%$ in minimum-bias $\mathrm{PbPb}$ collisions at LHC energies [31, 32,33] is thus due to damping and gluodissociation, and to the reduced feed-down from the suppressed excited states [30]. The subsequently outlined model $[34,30]$ aims to account for these processes.

We have not discussed explicitly the production mechanism of the bottomium states, but rather use initial populations as deduced from the experimental CMS results in $p p$ at the same center of mass energy [33], and a distribution according to the number of binary collisions. The final populations of the $\Upsilon(n S)$ states in $p p$ collisions are measured from $\mu^{+} \mu^{-}$decays, and we calculate the initial populations through an inverted decay cascade using the CMS $2.76 \mathrm{TeV}$ data for $\Upsilon(n S)$ and CDF $p \bar{p}$ data at $1.8 \mathrm{TeV}[35]$ for $\chi_{b}(n P)$.

The calculation of the $b \bar{b}$ wave functions for $\operatorname{six} \Upsilon(n S)$ and $\chi_{b}(n P)$ states, and the associated widths $\Gamma_{\text {damp }}$ of these states due to collisional damping from a complex potential are considered in the following section as detailed in [30]. Next the calculation of the gluodissociation decay widths $\Gamma_{\text {diss }}$ for the same states is discussed [34]. The time evolution of the fireball and subsequent decay cascade in $\mathrm{PbPb}$ at $\sqrt{s_{N N}}=2.76 \mathrm{TeV}$ is then considered, and the results are presented in comparison with the available CMS data at LHC energies. The conclusions are drawn in the last section.

\subsection{Bottomium wave functions}

Due to the small relative velocity $v \ll c$ of the bottom quarks, a nonrelativistic potential model is adequate for the description of bottomium states. This leads to a Schrödinger equation, with the coulombic, color-singlet potential $V=-C_{F} \alpha_{s}^{s} / r$. Here the strong coupling constant at the soft scale is $\alpha_{s}^{s}=\alpha_{s}\left(m_{b} \alpha_{s} / 2\right)=0.48$, and $N_{c}=3, C_{F}=\left(N_{c}^{2}-1\right) /\left(2 N_{c}\right)=4 / 3$. The bottom mass is $m_{b}=4.89 \mathrm{GeV}$.

In the QGP environment the calculation is made at finite temperature which yields for the short-range part of the potential, in the HTL approximation, a complex, screened, coulombic expression $[36,37]$ that we have used in our phenomenological approach [30]. The imaginary part 
of the potential causes a decay width $\Gamma_{\text {damp }}$, monotonically increasing with temperature, which accounts for collisional damping by the plasma particles.

This potential does, however, not yet contain the long-range non-perturbative string contribution which causes confinement and vanishes due to screening only at sufficiently high temperature $T>T_{c}$. Since a consistent derivation is not possible, we have parametrized the long-range part as in [38] so that the full singlet potential reads

$$
V\left(r, m_{D}\right)=\frac{\sigma}{m_{D}}\left(1-e^{-m_{D} r}\right)-\alpha_{e f f}\left(m_{D}+\frac{e^{-m_{D} r}}{r}\right)-i \alpha_{e f f} T \int_{0}^{\infty} \frac{d z 2 z}{\left(1+z^{2}\right)^{2}}\left(1-\frac{\sin \left(m_{D} r z\right)}{m_{D} r z}\right),
$$

with the Debye mass $m_{D}=T \sqrt{4 \pi \alpha_{s}^{T}\left(\frac{N_{c}}{3}+\frac{N_{f}}{6}\right)}, \alpha_{e f f}=4 \alpha_{s}^{s} / 3$, the number of flavors in the QGP $N_{f}=3$, and the strong coupling constant evaluated at the HTL energy $2 \pi T, \alpha_{s}^{T}=\alpha_{s}(2 \pi T) \leq 0.50$, respectively. The absolute values $\left|g_{n l}(r)\right|$ of the resulting $b \bar{b}$ wave functions as calculated in the complex screened potential are shown in [30].

The Schrödinger equation is now solved for every $b \bar{b}$ state with the potential (4.1) for $T \geq$ $T_{c}$ up to the dissociation temperature $T_{\text {diss }}$ above which screening prevents bottomium formation and no bound states solutions exist. The dissociation temperatures with the above parameters are $T_{\text {diss }} \simeq 668,217$ and $206 \mathrm{MeV}$ for $\Upsilon(1 S), \Upsilon(2 S)$ and $\chi_{b}(1 P)$, respectively: The higher excited states are already dissolved for $T>T_{C}$.

\subsection{Gluodissociation in the medium}

We have shown in [34] that at LHC energies, gluodissociation is a major process besides collisional damping and reduced feed-down that leads to a suppression of $\Upsilon$ 's. This is due to the high gluon density reached in the mid-rapidity region. Hence we have calculated in $[34,30]$ the gluodissociation cross sections for the $\Upsilon(1 S)-\Upsilon(3 S)$, and $\chi_{b}(1 P), \chi_{b}(2 P), \chi_{b}(3 P)$ states for different lifetimes $t_{Q G P}$ of the QGP.

Bhanot and Peskin (BP) had already derived long time ago the leading-order dissociation cross section of the $b \bar{b}$ states through E1 absorption of a single gluon [39]. On the other hand, the gluodissociation cross section may be obtained from the dipole interaction term describing a singlet-octet transition of the $b \bar{b}$-pair via emission/absorption of an ultra soft gluon. From this starting point we have generalized the approach to include the effect of our modified potential (4.1) [40], and obtain for a bottomium state $(n l)$ [30]

$$
\begin{gathered}
\sigma_{\text {diss }, n l}\left(E_{g}\right)=\frac{2 \pi^{2} \alpha_{s}^{u} E_{g}}{(2 l+1) N_{c}^{2}} \sum_{m=-l l^{\prime}=0}^{l} \sum_{m^{\prime}=-l^{\prime}}^{l^{\prime}} \cdot \int_{0}^{\infty} d q|<n l m| \hat{\vec{r}}\left|q l^{\prime} m^{\prime}>\right|^{2} \delta\left(E_{g}+E_{n l}-\frac{q^{2}}{m_{b}}\right) \\
=\frac{\pi^{2} \alpha_{s}^{u} E_{g}}{N_{c}^{2}} \sqrt{\frac{m_{b}}{E_{g}+E_{n l}}} \frac{l\left|J_{n l}^{q, l-1}\right|^{2}+(l+1)\left|J_{n l}^{q, l+1}\right|^{2}}{2 l+1}
\end{gathered}
$$

where $J_{n l}^{q l^{\prime}}=\int_{0}^{\infty} d r r g_{n l}^{*}(r) h_{q l^{\prime}}(r)$. The singlet and octet states are $|n l m>|, q l^{\prime} m^{\prime}>$ and $\alpha_{s}^{u}=\alpha_{s}\left(m_{b} \alpha_{s}^{2} / 2\right) \simeq$ 0.59. The radial wave function $h_{q l^{\prime}}$ of the states $\mid q l^{\prime} m^{\prime}>$ is derived from the octet Hamiltonian with the potential $V_{8}=+\alpha_{e f f} /(8 r)$, and the value of $q$ is as determined from energy conservation, $q=\sqrt{m_{b}\left(E_{g}+E_{n l}\right)}$. The use of the $\delta$-function is an approximation, the actual energy-conserving function in a complex potential acquires a width (Breit-Wigner distribution). 


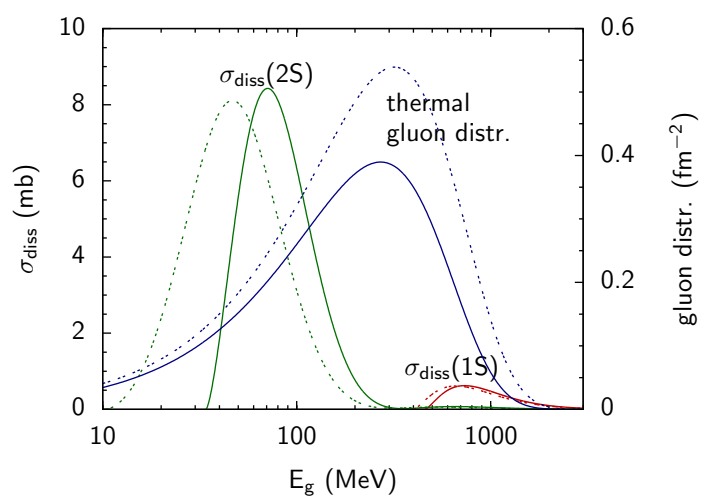

Figure 7: Gluodissociation cross sections $\sigma_{\text {diss }}(n S)$ in mb (left scale) of the $\Upsilon(1 S)$ and $\Upsilon(2 S)$ states calculated using the screened complex potential for temperatures $T=170$ (solid curves) and $200 \mathrm{MeV}$ (dotted curves) as functions of the gluon energy $E_{g}$. The thermal gluon distribution (right scale; solid for $T=170$ $\mathrm{MeV}$, dotted for $200 \mathrm{MeV}$ ) is used to obtain the thermally averaged cross sections through integrations over the gluon momenta. From [30].

The mean gluodissociation cross section, is then obtained by averaging our calculated gluodissociation cross sections over the Bose-Einstein distribution function of gluons at temperature $T$. We thus assume that the medium is thermalized, although the heavy $b \bar{b}$ is not (see figure 7 for the gluon distribution):

$$
\Gamma_{\text {diss }, n l}=\frac{g_{d}}{2 \pi^{2}} \int_{0}^{\infty} \frac{d p_{g} p_{g}^{2} \sigma_{d i s s, n l}\left(E_{g}\right)}{e^{E_{g} / T}-1},
$$

where $g_{d}=16$ is the number of gluonic degrees of freedom. This expression is valid for an idealized case of Upsilons at rest in a thermal bath of gluons with temperature T. However, produced quarkonia are never strictly at rest, but have an rms momentum of several GeV. We shall discuss this effect infuture work.

The total decay width in the QGP is obtained from the sum of the collisional decay width $\Gamma_{\text {damp }}$ together with the resulting width from gluodissociation, $\Gamma_{t o t}=\Gamma_{d a m p}+\Gamma_{d i s s}$. The partial widths $\Gamma_{\text {damp }}$ and $\Gamma_{\text {diss }}$ are calculated in [30]. Both processes are of the same order of magnitude and need to be considered when calculating the total width in the quark-gluon plasma. Whereas damping increases monotonically with temperature, gluodissociation reaches a maximum, and decreases again at very high temperatures beyond $600 \mathrm{MeV}$ due to the diminishing overlap of the thermal gluon distribution and the gluodissociation cross section at large values of $\mathrm{T}$.

\subsection{Expansion of the medium and feed-down}

The number $N_{b \bar{b}}$ of produced $b \bar{b}$-pairs at the point $(x, y)$ in the transverse plain and impact parameter $b$ is proportional to the number of binary collisions $N_{\text {coll }}$ and nuclear overlap $T_{A A}$, $N_{b \bar{b}}(b, x, y) \propto N_{\text {coll }}(b, x, y) \propto T_{A A}(b, x, y)$. The bottomium states are created at a formation time which is taken here as $t_{F}=0.1 \mathrm{fm} / c$, but will be investigated in more detail in future work. In [30], we have taken the initial temperature to be proportional to the number of collisions, and Bjorken scaling is used for the time evolution [41, 42], $T(b, t, x, y) \propto\left(\frac{t_{Q G P}}{t}\right)^{1 / 3}$, with $t_{Q G P}$ the maximum 


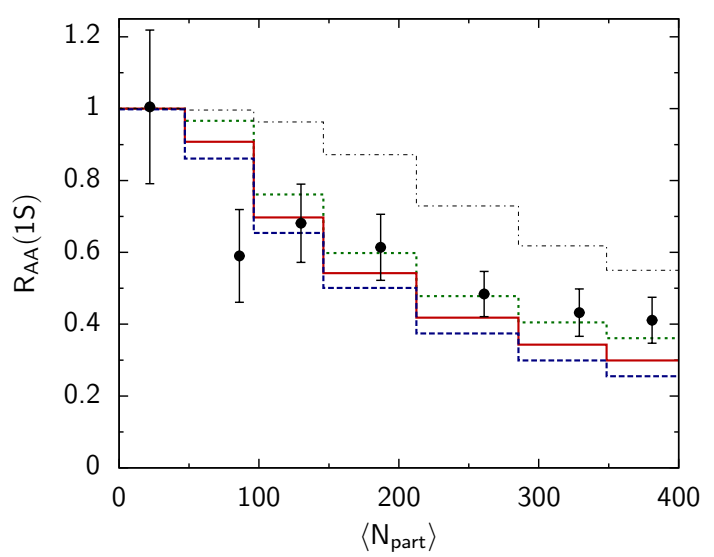

Figure 8: Suppression factor $R_{A A}$ for the $\Upsilon(1 S)$ ground state calculated for $2.76 \mathrm{TeV} \mathrm{PbPb}$-collisions from screening, collisional damping, gluodissociation and reduced feed-down using three QGP lifetimes $t_{Q G P}=4$, $6,8 \mathrm{fm} / c$ (dotted, solid and dashed line respectively) for the centrality bins 50-100\%, 40-50\%, 30-40\%, 20$30 \%, 10-20 \%, 5-10 \%, 0-5 \%$. The dash-dotted upper line is the QGP suppression factor $R_{A A}^{Q G P}(1 \mathrm{~S})\left(t_{Q G P}=6\right.$ $\mathrm{fm} / c$ ) without reduced feed-down. The corresponding CMS data [33] are in good agreement with the model results for the $\Upsilon(1 S)$ state. From [30].

lifetime of the quark-gluon plasma. We shall soon improve on these assumptions by investigating also different relations between the initial temperature distribution and the number of collisions and/or participants, and by including also the effect of transverse expansion in a hydrodynamic approach, which leads to additional cooling in the fireball.

The suppression factor in the quark-gluon plasma $R_{A A}^{Q G P}$, which accounts only for the $b \bar{b}$ suppression due to the three processes Debye screening, collisional damping and gluodissociation, can then be expressed as

$$
R_{A A}^{Q G P}=\frac{\int d^{2} b \int d x d y T_{A A}(b, x, y) e^{-\int_{t_{F}}^{\infty} d t \Gamma_{t o t}(b, t, x, y)}}{\int d^{2} b \int d x d y T_{A A}(b, x, y)}
$$

The $\Upsilon(2 S)$ is found to be suppressed much more efficiently than the more stable $\Upsilon(1 S)$. Also one should note the action of Debye screening which forbids the formation of bound $b \bar{b}$ states at sufficiently high temperatures.

Having calculated the suppression during the evolution of the fireball we have next considered the feed-down of the remaining $b \bar{b}$ population to calculate the fraction of decays into dimuon pairs, $\Upsilon(n S) \rightarrow \mu^{+} \mu^{-}$[30]. The results that are discussed in the next section will include the effect of the feed-down cascade.

\subsection{Results for bottomium suppression}

We have presented in [30] the results for screening and collisional damping derived from the solutions of the Schrödinger equation with the potential eq. (4.1), and the widths for gluodissociation as derived from eq. (4.3). The total decay widths $\Gamma_{t o t}$ are then inserted into a dynamic calculation for the fireball evolution to calculate QGP suppression factors, eq. (4.4). The bottomium states pass through a decay cascade (see [43]) so that the higher excited states feed the lower lying states to yield the final suppression factor. 


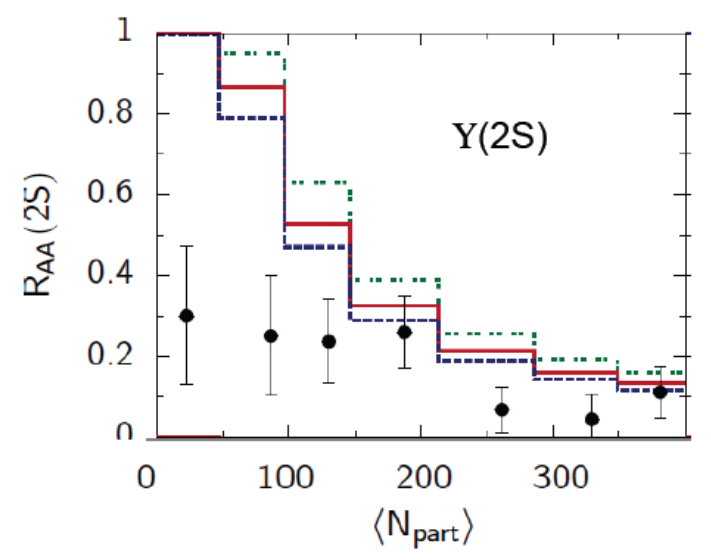

Figure 9: Suppression factor $R_{A A}$ for the $\Upsilon(2 S)$, state calculated for $2.76 \mathrm{TeV} \mathrm{PbPb}$-collisions from screening, collisional damping, gluodissociation and feed-down using three QGP lifetimes $t_{Q G P}=4,6,8 \mathrm{fm} / \mathrm{c}$ (dotted, solid and dashed line respectively) for seven centrality bins The corresponding CMS data [33] for the $\Upsilon(2 S)$ state show significantly more suppression, in particular, in the peripheral region. From [30].

Results for the suppression of the $\Upsilon(1 S)$ state in $\mathrm{PbPb}$ relative to $p p$ are shown in figure 8 [30] for three different QGP lifetimes $t_{Q G P}=4,6,8 \mathrm{fm} / c$ as functions of centrality (number of participants). When comparing with our result from the QGP suppression factor (upper dotted step function), it is evident that the consideration of the feed-down cascade is essential for modeling the suppression. The calculated suppression is obviously in very good agreement with the CMS data for the $\Upsilon(1 S)$ ground state. This is also true for minimum bias (centrality integrated) results.

The calculated suppression factors depend rather strongly on the Upsilon formation time. Shorter formation times cause more suppression in the QGP, because the dissociation processes start to act at a higher initial temperature and hence, are more efficient. Typical results for the suppression of $\Upsilon(1 S)$ in minimum bias collisions with gluodissociation and damping for a QGP lifetime of $6 \mathrm{fm} / \mathrm{c}$ are $R_{A A}(1 S)=0.74,0.63$ and 0.45 for $t_{F}=1,0.5$ and $0.1 \mathrm{fm} / c$, respectively.

Results for the suppression of the $\Upsilon(2 S)$ state in $\mathrm{PbPb}$ relative to $p p$ are shown in figure 9 [30] for three different QGP lifetimes $t_{Q G P}=4,6,8 \mathrm{fm} / c$ as functions of centrality, and compared with CMS data [33].

The suppression found experimentally for the $\Upsilon(2 S)$ state is much more pronounced than in the calculation, in particular, for the three more peripheral data points. In future work we shall modify the assumptions that enter the model in order to better understand the relatively strong suppression already in very peripheral collisions. It should be noted that other theoretical approaches such as $[44,45,46]$ also find that the $\Upsilon(2 S)$ suppression factor rises towards 1 for peripheral collisions, in contrast with the data.

\section{Conclusions}

In the QCD-based treatment of stopping in relativistic heavy-ion collisions, the fragmentation peaks occur due to the interaction of valence quarks with gluons. The qualitatively different dependence of the fragmentation peak position, and the mean rapidity loss on the beam rapidity offers a clear indication for the onset of hadronic behaviour - confinement - at low energies. 
The transition from partonic to hadronic behaviour in central collisions as the beam energy decreases is gradual, there is no sudden deconfinement with a corresponding jump in the mean rapidity loss at a particular beam energy. Hadronic and partonic processes coexist in a large energy region that extends from AGS to low LHC energies. In the fragmentation peak region, partonic processes are decisive even at low SPS energies.

Regarding particle production in the nonequilibrium-statistical RDM approach, I have determined the particle content of fragmentation (valence quark - gluon) and midrapidity (gluon gluon) sources for charged-hadron production in heavy-ion collisions at high relativistic energies as function of c.m. energy. In turns out that particle production from the gluon-gluon source becomes more important than that from the fragmentation sources in the energy range between the maximum RHIC energy of $0.2 \mathrm{TeV}$, and the current LHC energy of $2.76 \mathrm{TeV}$.

Our phenomenological model for Upsilon suppression in $\mathrm{PbPb}$ collisions at LHC energies yields a reasonable description of the ground state suppression due to gluodissociation, damping, and reduced feed-down, although there are caveats related to various model assumptions. Screening is unimportant for the $\Upsilon(1 S)$ state. For the excited states the model reveals substantial screening effects and - together with the other dissociation processes that we consider - larger suppression than for $\Upsilon(1 S)$, but it disagrees quantitatively with the current CMS data regarding the centrality dependence. Hence there is considerable room for future improvement.

\section{Acknowledgments}

I am grateful to former graduate students Rolf Kuiper and David Röhrscheid for their participation in the RDM calculations, to Yacine Mehtar-Tani for our collaboration in the description of stopping, to Felix Nendzig for his contribution to the Upsilon project in his $\mathrm{PhD}$ thesis, as well as to BSc students Johannes Wahner, Francesco Vaccaro and Thilo Kind in the same context.

\section{References}

[1] G. Wolschin, Prog. Part. Nucl. Phys. 59, 374 (2007)

[2] Y. Mehtar-Tani, G. Wolschin, Phys. Rev. Lett. 102, 182301 (2009)

[3] Y. Mehtar-Tani, G. Wolschin, Phys. Rev. C 80, 054905 (2009)

[4] H. Appelshäuser et al. (NA49 Collaboration), Phys. Rev. Lett. 82, 2471 (1999)

[5] C. Blume et al. (NA49 Collaboration), PoS (Confinement) 8, 110 (2008)

[6] I.G. Bearden et al. (BRAHMS Collaboration), Phys. Rev. Lett. 93, 102301 (2004)

[7] H.H. Dalsgaard et al. (BRAHMS Collaboration), Int. J. Mod. Phys. E 16, 1813 (2007)

[8] R. Debbie et al. (BRAHMS Collaboration), J. Phys. G 35, 104004 (2008)

[9] I.C. Arsene et al. (BRAHMS Collaboration), Phys. Lett. B 677, 267 (2009)

[10] Y. Mehtar-Tani, G. Wolschin, EPL 94, 62003 (2011)

[11] G. Wolschin, Prog.Theor.Phys.Suppl. 193, 220 (2012)

[12] F. Videbaek, O. Hansen, Phys. Rev. C 52, 2684 (1995) 
[13] C. Blume et al. (NA49 Collaboration), J. Phys. G 34, 951 (2007)

[14] J.L. Albacete, Phys. Rev. Lett. 99, 262301 (2007)

[15] I.G. Bearden et al. (BRAHMS Collaboration), Phys. Rev. Lett. 87, 112305 (2001)

[16] G. Wolschin, J. Phys. G: Nucl. Part. Phys. 40, 045104 (2013)

[17] G. Wolschin, Eur. Phys. J. A 5, 85 (1999)

[18] G. Wolschin, Europhys. Lett. 47, 30 (1999)

[19] G. Wolschin, M. Biyajima, T. Mizoguchi, N. Suzuki, Phys. Lett. B 633, 38 (2006)

[20] D. Röhrscheid, G. Wolschin, Phys. Rev. C 86, 024902 (2012)

[21] R. Kuiper, G. Wolschin, EPL 78, 2201 (2007)

[22] B. Alver et al. (PHOBOS Collaboration), Phys. Rev. C 83, 024913 (2011)

[23] K. Aamodt et al. (ALICE Collaboration), Phys. Rev. Lett. 106, 032301 (2011)

[24] M. Guilbaud et al. (ALICE Collaboration), Nucl. Phys. A 904-905, 381c (2013)

[25] J. Benecke, T. Chou, C. Yang, E. Yen, Phys. Rev. 188, 2159 (1969)

[26] T. Matsui, H. Satz, Phys. Lett. B 178, 416 (1986)

[27] B.K. Patra, D.K. Srivastava, Phys. Lett. B 505, 113 (2001)

[28] D.E. Kharzeev, J. Phys. G: Nucl. Part. Phys. 34, S445 (2007)

[29] L. Kluberg, H. Satz, Landolt Börnstein 23, 373 (2010)

[30] F. Nendzig, G. Wolschin, Phys. Rev. C 87, 024911 (2013)

[31] S. Chatrchyan, V. Khachatryan, A.M. Sirunyan, A. Tumasyan, W. Adam, T. Bergauer, M. Dragicevic, J. Erö, C. Fabjan, M. Friedl et al., Phys. Rev. Lett. 107, 052302 (2011)

[32] S. Chatrchyan et al. (CMS Collaboration), JHEP 1205, 063 (2012)

[33] S. Chatrchyan et al. (CMS Collaboration), Phys. Rev. Lett. 109, 222301 (2012)

[34] F. Brezinski, G. Wolschin, Phys. Lett. B 707, 534 (2012)

[35] T. Affolder, H. Akimoto, A. Akopian, M.G. Albrow, P. Amaral, S.R. Amendolia, D. Amidei, J. Antos, G. Apollinari, T. Arisawa et al., Phys. Rev. Lett. 84, 2094 (2000)

[36] M. Laine, O. Philipsen, M. Tassler, P. Romatschke, JHEP 0703, 054 (2007)

[37] A. Beraudo, J.P. Blaizot, C. Ratti, Nucl. Phys. A 806, 312 (2008)

[38] F. Karsch, M.T. Mehr, H. Satz, Z. Phys. C 37, 617 (1988)

[39] G. Bhanot, M.E. Peskin, Nucl. Phys. B 156, 391 (1979)

[40] F. Nendzig, Ph.D. thesis, University of Heidelberg (2013), in preparation

[41] J. Bjorken, Phys. Rev. D 27, 140 (1983)

[42] K. Yagi, T. Hatsuda, Y. Miake, Quark-Gluon Plasma (Cambridge University Press, Cambridge, UK, 2008)

[43] F. Vaccaro, F. Nendzig, G. Wolschin, EPL 102, 42001 (2013)

[44] T. Song, K.C. Han, C.M. Ko, Phys. Rev. C 85, 014902 (2012)

[45] A. Emerick, X. Zhao, R. Rapp, Eur. Phys. J. A 48, 72 (2012)

[46] M. Strickland, D. Bazow, Nucl. Phys. A 879, 25 (2012) 